\title{
APLICABILIDADE DA FOLKSONOMIA NA CONSTRUÇÃO E ATUALIZAÇÃO DE SISTEMAS HÍBRIDOS DE ORGANIZAÇÃO DO CONHECIMENTO
}

Resumo: Estuda aspectos relativos à Folksonomia e à representação colaborativa da informação em Sistemas de Recuperação da Informação. Apresenta as suas principais características, implicações positivas e negativas no processo de representação e recuperação da informação. Tem como objetivo geral analisar a aplicabilidade da Folksonomia no processo de construção e atualização de Sistemas híbridos de Organização do Conhecimento, a partir dos resultados de pesquisas nacionais e internacionais na literatura. Utiliza como metodologia as pesquisas bibliográfica, exploratória, descritiva com abordagem qualitativa. Evidencia os principais contributos das ferramentas que combinam a estrutura sistematizada dos instrumentos tradicionais de representação do conhecimento à semântica latente da Folksonomia em Sistemas de Recuperação da Informação. Apresenta propostas que exploram as potencialidades da Folksonomia com o suporte dos formalismos e métodos utilizados na construção de instrumentos de controle terminológicos, descortinando novas perspectivas para auxiliar a representação e a troca de conhecimentos em ambiente web.

Palavras-chave: Folksonomia; representação do conhecimento; organização do conhecimento; sistemas híbridos de organização do conhecimento.
Raimunda Fernanda dos Santos Doutora em Ciência da Informação pela Universidade Federal da Paraíba (UFPB). Docente do Departamento de Biblioteconomia da Universidade

Federal do Rio de Janeiro (UFRJ). fernanda@facc.ufrj.br

\section{Maria Elizabeth Baltar Carneiro de} Albuquerque

Doutora em Letras pela Universidade Federal de João Pessoa (UFPB). Professora Titular do Departamento de Ciência da Informação e do Programa de Pós-graduação em Ciência da Informação da UFPB. ebaltar2007@gmail.com

\section{APPLICABILITY OF FOLKSONOMY IN THE CONSTRUCTION AND UPDATE OF HYBRID KNOWLEDGE ORGANIZATION SYSTEMS}

\begin{abstract}
Studies aspects related to Folksonomy and the collaborative representation of information in Information Retrieval Systems. Presents its main characteristics, positive and negative implications in the process of representation and information retrieval. General objective is to analyze the applicability of Folksonomy in the process of building and updating hybrid Knowledge Organization Systems, based on the results of national and international research in the literature. Uses bibliographic, exploratory, descriptive research with a qualitative approach as a methodology. Highlights the main contributions of the tools that combine the systematized structure of traditional knowledge representation tools with the latent semantics of Folksonomy in Information Retrieval Systems. Presents proposals that explore the potential of Folksonomy with the support of formalisms and methods used in the construction of terminological control instruments, revealing new perspectives to help the representation and exchange of knowledge in a web environment.
\end{abstract}

Keywords: Folksonomy; Knowledge Representation; Knowledge Organization; Hybrid Knowledge Organization Systems.

\section{INTRODUÇÃO}

Com o desenvolvimento da Web, o barateamento e o acesso aos computadores pessoais e dispositivos móveis, surge uma explosão de dados gerados por usuários conectados em todos 
os lugares, a cada minuto por câmeras de monitoramento, GPS, fotos, vídeos, aplicativos, posts, e-mails, mensagens, entre outros, gerando uma produção exorbitante de dados.

Esse fenômeno foi conceituado como Big Data, que diz respeito aos conjuntos de dados que estão além da capacidade de captura, armazenamento e análise das ferramentas tradicionais de software (MANYIKA et al., 2011).

Com o advento de aplicações da web que promovem a participação colaborativa e ativa dos usuários, as atividades de representação de conteúdos informacionais nesse contexto têm sido executadas por sujeitos e máquinas por meio da Folksonomia. Através dela os usuários (humanos ou robôs) assumem o protagonismo nas práticas de descrição e recuperação dos objetos informacionais em ambientes colaborativos, possibilitando que, através da linguagem natural, sejam selecionadas e atribuídas as palavras-chave consideradas mais pertinentes para descrever os itens informacionais em diferentes formatos, sejam eles áudios, textos, imagens, vídeos etc.

Nessa perspectiva, de acordo com Corrêa e Santos (2018) a Folksonomia pode ser conceituada como o resultado da atribuição livre de palavras-chave realizada por usuários (humanos ou robôs), sem o auxílio de instrumentos de controle terminológico, em sistemas colaborativos para fins de representação e recuperação de objetos informacionais em diferentes formatos, sejam eles áudios, textos, imagens, vídeos etc.

Através da Folksonomia é possível que usuários (humanos ou robôs) atribuam etiquetas ou tags aos recursos informacionais que julgam melhor representar as informações contidas nesses itens. Essa prática possibilita a aproximação entre os usuários e os itens informacionais, a ampliação das possibilidades de encontrabilidade ${ }^{1}$ e serendipidade ${ }^{2}$ além de contribuir para a busca e navegação, por meio de tags associadas às características e às especificidades desses recursos.

A Folksonomia vem se configurando em um campo de estudo ainda a ser explorado na Organização e Representação do Conhecimento e na Ciência da Informação, sobretudo no que diz respeito às diversas ferramentas, modelos, serviços, sistemas, instrumentos e aplicações em que ela pode estar inserida ou vinculada.

\footnotetext{
${ }^{1}$ Vocábulo proveniente do termo em inglês 'findability', diz respeito à qualidade de um objeto informacional ser localizável ou navegável em um Sistema de Recuperação da Informação (MORVILLE, 2005).

${ }^{2}$ Experiências de descoberta fortuitas de informação (SOLOMON; BOSTEIN, 2016).
} 
Os usuários atribuem sentido às coisas pela maneira com as quais utilizam ou as integram e associam em suas práticas cotidianas; bem como pela forma que as representam por meio das palavras empregadas que, posteriormente, são classificadas e conceituadas. Nessa perspectiva, concorda-se com Limpens, Gandon e Buffa (2009), quando os mesmos ressaltam que a Folksonomia consiste em um sistema colaborativo de representação do conhecimento que contempla uma estrutura triádica: etiquetas, pessoas e recursos web.

Diante desse cenário, acredita-se que o Big Data e os ambientes colaborativos não eliminarão a necessidade e as contribuições dos tradicionais Sistemas de Organização do Conhecimento, haja vista que comunidades virtuais fazem grandes contribuições para o compartilhamento do conhecimento. No entanto, as transformações em curso citadas anteriormente e decorrentes desse fenômeno pressionam a comunidade da área de Organização e Representação do Conhecimento a refletir acerca do ponto de vista em que os Sistemas de Organização do Conhecimento tradicionais são criados, demandando a necessidade de se adaptar ao novo mundo do conhecimento a partir de esquemas, sistemas e instrumentos híbridos e flexíveis.

Nesse entendimento, acredita-se que a Folksonomia pode contribuir para a construção, atualização e hibridização de Sistemas de Organização do Conhecimento, ao mesmo tempo em que também pode ser enriquecida semanticamente por instrumentos de controle terminológico em sistemas colaborativos.

Essa cooperação resulta no que denominamos nesta pesquisa de sistemas híbridos de organização do conhecimento, os quais agregam as potencialidades das Folksonomias e de outro(s) Sistemas de Organização do Conhecimento (ontologia, taxonomia, tesauro, por exemplo), podendo ser aplicados em domínios específicos, objetivando a produção significativa de metadados semânticos para fins de representação e recuperação de conteúdos na web.

Diante do exposto, esta investigação tem como objetivo geral analisar a aplicabilidade da Folksonomia no processo de construção e atualização de Sistemas híbridos de Organização do Conhecimento, a partir dos resultados de pesquisas nacionais e internacionais na literatura.

Para o desenvolvimento deste estudo foram realizadas as pesquisas bibliográfica, exploratória e descritiva com abordagem qualitativa a partir da coleta e análise dos aspectos enfatizados nas produções científicas nacionais e internacionais sobre Folksonomia e a sua integração com Sistemas de Organização do Conhecimento (como ontologias, taxonomias e 
tesauros, por exemplo) nas seguintes fontes de informação: Portal de Periódicos CAPES; Base de Dados em Ciência da Informação (BRAPCI) e Biblioteca Digital Brasileira de Teses e Dissertações (BDTD). As produções científicas recuperadas nessas fontes que se articulavam diretamente com o objetivo deste estudo foram citadas como referencial teórico com o objetivo de apresentar ideias e reflexões que possam colaborar com as contribuições aqui apresentadas.

A relevância desta pesquisa decorre, em linhas gerais, das novas possibilidades de representação do conhecimento em ambientes digitais e uma das principais justificativas para a realização deste estudo está associada à incipiência de produções científicas sobre o tema em questão na literatura brasileira da Organização do Conhecimento e da Ciência da Informação.

Seguindo essa linha de raciocínio, a seguir são apresentadas questões concernentes aos Sistemas de Organização do Conhecimento e aos princípios que servem como base para a construção e atualização de instrumentos de controle terminológico, os quais carecem de ser estudados sob o viés das práticas folksononômicas enquanto representação social do conhecimento dos indivíduos.

\section{SISTEMAS DE ORGANIZAÇÃO DO CONHECIMENTO}

Os Sistemas de Organização do Conhecimento (SOCs), denominados originalmente por Knowledge Organization Systems (KOS), se configuram como instrumentos cujos esquemas representam o conhecimento. Esses sistemas se diferenciam a partir de suas estruturas, relacionamentos entre termos, funções e complexidade.

Com a ampliação da quantidade de sistemas de recuperação da informação e o incremento de tecnologias aplicadas às atividades de representação, organização, recuperação, acesso e uso de conteúdos informacionais, surgiram diferentes Sistemas de Organização do Conhecimento para além das classificações amplamente conhecidas.

Zeng (2008) apresenta uma lista desses Sistemas de Organização do Conhecimento por meio das suas tipologias, a saber:

\section{Listas de Termos}

- $\quad \underline{\text { Listas }}$ propriamente ditas (pick lists): conjunto de termos em ordem sequencial;

- Dicionários: listas de termos organizados alfabeticamente com as suas respectivas definições, apresentando significados variados de acordo com a aplicação desses termos; 
- Glossários: listas de termos organizados alfabeticamente com as suas respectivas definições;

- Anéis de sinônimos: conjunto de descritores considerados como equivalentes para fins de representação e recuperação da informação.

\section{Modelos semelhantes a metadados:}

- Arquivos de autoridade: listas de termos empregados para fins de controle das variações de nomes para uma autoridade;

- Diretórios: listas de nomes e informações de contato associadas;

- Gazetteers: dicionários geoespaciais de tipos e nomes de lugares.

Classificação e categorização:

- Cabeçalhos de assunto: esquemas que apresentam um conjunto de descritores controlados para representar o assunto dos itens em uma coleção e dispõem um conjunto de diretrizes para combinar descritores em cabeçalhos compostos;

- Esquemas de categorização: esquemas de agrupamento "vagamente formados" (ZENG, 2008, p. 161);

- Taxonomias: separação de itens em grupos ordenados ou categorias, considerando características particulares;

- Esquemas de classificação: arranjos hierárquicos e facetados que apresentam notações alfabéticas ou numéricas visando representar assuntos genéricos.

$\checkmark$ Modelos de relações:

- Tesauro: listas de termos autorizados de um domínio específico do conhecimento, constituída de base léxica composta por termos que apresentam relações hierárquicas, de equivalência e associativas entre eles.

- Redes semânticas: conjunto de descritores modelados como nós em uma rede de variados tipos de relações objetivando representar conceitos;

- Ontologias: modelos de conceitos específicos que representam relações complexas entre objetos, incluindo regras e axiomas ausentes em redes semânticas.

Em posição complementar, Soler Monreal e Gil Leiva (2010) ilustram algumas dessas tipologias, que parte das mais simples até as mais complexas, através da linha progressiva ascendente apresentada na Figura 1. 
Figura 1: Estruturas e estratégias para a organização do conhecimento

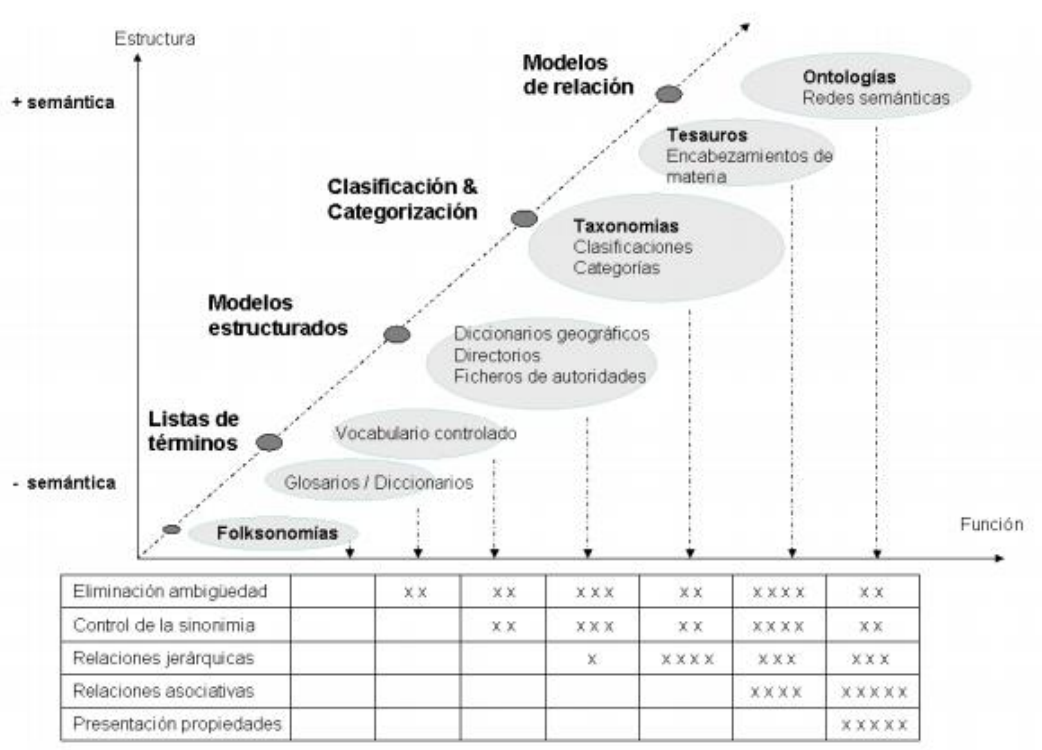

Fonte: Soler Monreal e Gil Leiva (2010, p. 364).

A Figura 1 elenca os graus de formalização semântica dos descritores apresentados nos Sistemas de Organização do Conhecimento, a partir das suas respectivas características funcionais e estruturais. Como é possível visualizar na ilustração, a Folksonomia aparece como um SOC de baixo grau de formalização semântica e as ontologias aparecem em posição contrária, tendo em vista que essas últimas consistem em sistemas complexos que sustentam as bases de conhecimento das aplicações web.

Considerando que as funções dos SOC diversificam conforme o grau de complexidade e o objetivo de sua aplicação, Moreira (2018) também representa em sua pesquisa a complexidade desses sistemas:

Figura 2: Complexidade dos SOC

\begin{tabular}{|c|c|c|c|}
\hline folksonomia & taxonomia & tesauro & ontologia \\
\hline menos & \multicolumn{2}{|c|}{ complexidade } & ma \\
\hline & $\begin{array}{l}\text { controle de } \\
\text { sinônimos }\end{array}$ & $\begin{array}{l}\text { controle de } \\
\text { sinónimos }\end{array}$ & $\begin{array}{l}\text { controle de } \\
\text { sinônimos }\end{array}$ \\
\hline & $\begin{array}{c}\text { relaçōes } \\
\text { hierárquicas }\end{array}$ & $\begin{array}{c}\text { relaçōes } \\
\text { hierárquicas }\end{array}$ & $\begin{array}{c}\text { relaçōes } \\
\text { hierárquicas }\end{array}$ \\
\hline & & $\begin{array}{c}\text { relaçōes } \\
\text { associativas }\end{array}$ & $\begin{array}{c}\text { relaçōes } \\
\text { associativas }\end{array}$ \\
\hline & & & $\begin{array}{c}\text { apresentação } \\
\text { de } \\
\text { propriedades }\end{array}$ \\
\hline
\end{tabular}

Fonte: Moreira (2018, p. 102).

PontodeAcesso, Salvador, v. 15, n. 3, p. 381-397, dez. 2021 
Nas Figuras 1 e 2, é possível verificar a inclusão da Folksonomia, uma vez que a lista de tipologias de SOC não é fechada e pode contemplar outras estratégias que viabilizam novas possibilidades de representação do conhecimento geradas pelos laços cognitivos e interações entre diferente sujeitos. Além disso, é importante ressaltar a viabilidade capturar a semântica na Folksonomia, a seção a seguir demonstra estudos que desenvolveram ontologias e taxonomias a partir da Folksonomia, por exemplo.

Não obstante, as vantagens dos instrumentos de controle terminológico sobressaiam em relação à linguagem natural, em algumas situações a aplicação dessa última espécie de linguagem se faz apropriada mediante a aplicação dos métodos e sistemas colaborativos de representação do conhecimento. A aplicação da linguagem natural se torna viável, sobretudo, para representar conteúdos complexos e altamente subjetivos que viabilizam variadas interpretações de grupos de sujeitos que possuem interesses, objetivos ou práticas em comum. Nessa perspectiva, a Folksonomia possui capacidade de representar explicitamente vozes minoritárias sociais e culturais de conhecimento histórico, além de fomentar a exaustividade e a especificidade da indexação e, consequentemente, recuperação da informação. Todavia, considera-se a importância de um tratamento linguístico mínimo das etiquetas sugeridas pelos usuários na atividade de representação do conhecimento em domínios especializados.

Os SOCs, de maneira geral, tornaram-se instrumentos essenciais em diferentes contextos e aplicações, uma vez que todo contexto (seja ele físico ou digital) depende e utiliza bases de conhecimento e narrativas com múltiplas intencionalidades para representar e recuperar objetos informacionais.

As garantias são componentes que subsidiam a compreensão, a construção e a atualização dos Sistemas de Organização do Conhecimento e contemplam um conjunto de preceitos para a validação e inclusão de consensos semânticos nesses sistemas.

Com isso, verifica-se na literatura a preocupação em ampliar, por meio de perspectivas interdisciplinares, a noção de garantia para diversos elementos teórico-metodológicos que contribuem para a organização e representação do conhecimento de maneira colaborativa. Afinal, a noção de garantia pode ser associada a uma “[...] lente através da qual podemos avaliar na medida em que uma classificação tem sentido e utilidade para os propósitos para os quais foi criada” (KWASNIK, 2010, p. 107, tradução nossa). 
Os aspectos relacionados à cultura e à semântica também assumem relevância no contexto das garantias, principalmente quando se trata de discussões sobre valores compartilhados, memória coletiva e unidades terminológicas em ambientes colaborativosconsiderando que indivíduos que fazem parte de diferentes culturas necessitam de diferentes tipos de acesso à informação.

Segundo Beghtol (1986) o termo "garantia cultural" foi cunhado por J. M. Lee ao discutir a visão de que "a base semântica ou categorial de uma dada classificação é o produto da cultura que a produziu". A garantia semântica, por sua vez, tem como objetivo orientar o desenvolvedor a verificar, justificar e validar os significados das unidades terminológicas em classes e conceitos de um SOC para uma determinada finalidade ou audiência (GUEDES, 2016).

Esse aspecto de complexidade e, ao mesmo tempo, de diversidade está associado às variadas significações, interpretações, representações existentes na própria cultura, pois para Hall (2016, p. 20) “em toda cultura há sempre uma grande variedade de significados a respeito de qualquer tema e mais de uma maneira de representá-lo ou interpretá-lo", tendo em vista que a cultura se relaciona a conceitos, ideias, sentimentos, emoções e senso de pertencimento.

De certa forma, "a garantia cultural introduz o fator ético na Organização do Conhecimento" (BARITÉ, 2011, p. 6, tradução nossa). Esse fator se torna elemento importante na atualidade, dada a intensificação do contato entre as culturas, o qual é facilitado pelas Tecnologias de Informação e Comunicação. Com isso, para fins de construção e atualização de Sistemas de Organização do Conhecimento, se faz necessário refletir acerca dos desafios éticos que se tornam, gradativamente, socialmente mais complexos.

Nesse limiar, Olson (2001, p. 659) propõe “[...] a adoção de uma postura ativa criandose espaços em nossos limites para as vozes dos que foram excluídos”. Assim, metodologias, técnicas e instrumentos que façam com que os sistemas de informação sejam mais acessíveis e colaborativos podem corroborar para o desenvolvimento de relações éticas nesse contexto.

Essa proposição pode ser justificada pelo fato de que, atualmente os usuários ainda se “deparam com dispositivos coercitivos na prática bibliotecária [ou de profissionais da informação] que limitam o acesso e uso da informação", os quais suprimem a verdadeira relação do usuário com o conhecimento (AQUINO; SANTANA, 2013). Esses dispositivos, por sua vez, estão vinculados às práticas de organização e representação do conhecimento e, em 
determinados casos, podem implicar negativamente na comunicação entre a linguagem dos usuários e a linguagem dos sistemas de recuperação da informação.

Seguindo essa linha de raciocínio, Santos e outros (2017) enfatizam que essas reflexões se mostram imprescindíveis às perspectivas de construção de Sistemas de Organização do Conhecimento, considerando a necessidade de buscar uma linguagem que não seja excludente e questione modelos de classificação cuja inflexibilidade impede que se represente a diversidade de maneiras menos díspares.

Para dar segmento a essas considerações, a seção a seguir dedica-se a discutir questões relativas à Folksonomia e representação colaborativa do conhecimento.

\section{FOLKSONOMIA E REPRESENTAÇÃO COLABORATIVA DO CONHECIMENTO}

A Folksonomia faz parte de uma terminologia cunhada em 2004 por Thomas Vander Wal com a junção das palavras "Folk" (do germânico: "povo", "grupo de pessoas") e "taxonomy" (do grego: "ciência ou técnica de classificação"). De acordo com Quintarelli (2005), o termo em si nasceu na busca de nomear uma atividade já implementada em ferramentas de sites como o Delicious e o Flickr.

O interesse pelas investigações sobre esse tema despontou em escolas de informação por meio de ensino e pesquisa com ênfase tecnológica, principalmente em institutos e faculdades de Ciência da Informação alemães e norte-americanos.

As falhas dos sistemas que utilizam a Folksonomia decorrem, em linhas gerais, dos erros ortográficos, das tags excessivamente personalizadas, da ambiguidade terminológica em etiquetas que representam os materiais informacionais e, mais recentemente, do uso de chatbots programados para atribuírem tags que propagam a rápida popularização de fake news (notícias falsas) na rede.

Por outro lado, se bem aplicada e mediada, a Folksonomia pode trazer significativas contribuições para as práticas de representação e recuperação de conteúdos em ambientes digitais, dentre as quais: auxílio no fornecimento de detalhes nas descrições dos objetos informacionais (imagens, textos, áudios, vídeos etc.); apoio na construção e atualização de instrumentos de controle terminológico para a representação e recuperação da informação. 
Na concepção de Mika (2005), as tags se configuram como entidades que referenciam a representação colaborativa do conhecimento entre os atores sociais, mediante o compartilhamento de narrativas, linguagens e signos comuns.

Umas das principais diferenças entre a Folksonomia e os vocabulários controlados consiste na inexistência de relações hierárquicas entre os termos, podendo os mesmos serem apresentados em conjunto no âmbito de um sistema colaborativo. Dessa maneira, as Folksonomias consistem em um sistema colaborativo de organização do conhecimento que apresenta fragilidade em relação ao grau de formalização da linguagem, mas que possui semântica implícita contribuindo para a encontrabilidade em um domínio de especialidade.

Segundo Mathes (2004), a Folksonomia também contribui para a serendipidade, ou seja, a descoberta casual de informações em ambientes digitais, a partir dos links gerados entre as tags, os quais contribuem para o processo de navegação em ambientes colaborativos.

Nesse limiar, a Folksonomia pode ser compreendida como um sistema democrático, descentralizado e inclusivo, uma vez que agregam e consideram o vocabulário de todas as pessoas, de todas as vozes e de diferentes pontos de vista, viabilizando variados pontos de acesso às informações (QUINTARELLI, 2005). Golder e Huberman (2005) complementam que a Folksonomia pode ser vista como um sistema alternativo e mais leve de classificação, uma vez que a finalidade de uma tag é organizar os registros do conhecimento representados pelos autores desses recursos.

A pesquisa realizada por Adler (2009) revela duas características importantes da representação colaborativa do conhecimento: a) a existência de um grau de consenso por parte dos usuários quanto à etiquetagem dos recursos; b) alcance visível e negociável das expressões de vozes minoritárias nos ambientes colaborativos, uma vez que as tags atribuídas são estabelecidas pelas convenções sociais da linguagem e da ideologia dos usuários.

A Folksonomia é construída em comunidades discursivas, uma vez que estão inseridas em ambientes cujos grupos de indivíduos compartilham colaborativamente conhecimentos, bem como possuem interesses, objetivos ou práticas em comum. Portanto, ela reflete as relações entre a linguagem e a comunidade, resultando em práticas e vocabulários diferentes daqueles presentes em discursos autorizados e normalizados (como os tesauros, as taxonomias e as ontologias, por exemplo). 
Em consonância de evidências, Yedid (2013) evidencia que a Folksonomia reflete o vocabulário da comunidade de usuários e contribuem para a identificação de tendências terminológicas entre atores sociais que descrevem um corpus de documentos ou itens específicos, podendo ser analisadas para selecionar termos preferidos dessas fontes, bem como criar ou atualizar instrumentos de controle terminológico.

Spiteri (2007) realizou um estudo em três sites (delicious, Furl e Technorati), na oportunidade constatou que as etiquetas analisadas nesses ambientes encontravam-se articuladas com uma quantidade significativa de orientações da National Information Standards Organization (NISO) para a construção, desenvolvimento e gerenciamento de vocabulários controlados - sobretudo em relação aos tipos de conceitos expressos, a predominância das tags e substantivos únicos, bem como o uso de ortografia reconhecida.

Como mencionado anteriormente, a sinergia das Folksonomias e dos instrumentos de controle terminológico pode apresentar benefícios para os sistemas colaborativos de representação e recuperação da informação na web semântica. Essa cooperação pode ser utilizada na construção de sistemas híbridos de organização do conhecimento, a fim de construir colaborativamente representações com base na linguagem da comunidade de usuários de domínios específicos, considerando os preceitos estabelecidos nas garantias apresentadas nesta pesquisa.

\subsection{SISTEMAS HÍBRIDOS DE ORGANIZAÇÃO DO CONHECIMENTO}

Dada a versatilidade e capacidade da Folksonomia para integrar outros vocabulários, as propostas existentes na literatura nacional e internacional recuperadas e analisadas evidenciam a necessidade de conciliar as bases folksonômicas com as estruturas de instrumentos de controle terminológico. As proposições, em sua maioria, objetivam apoiar as interações sociais e auxiliar o ciclo de vida das Folksonomias e ontologias, enquanto sistemas que sustentam as bases de conhecimento das aplicações web.

Essas propostas são denominadas nesta pesquisa de Sistemas híbridos de Organização do Conhecimento, considerando a capacidade e versatilidade das Folksonomias para integrar outros Sistemas de Organização do Conhecimento, bem como da possibilidade de ser enriquecida semanticamente por instrumentos como ontologias e taxonomias. 
O primeiro passo registrado na literatura por pesquisadores para processar dados extraídos das Folksonomias para a construção de instrumentos de controle terminológico tem sido calcular a similaridade de tags e inferir as relações semânticas entre etiquetas.

Mika (2005), por exemplo, realiza a extração de relações mais amplas e estreitas entre as tags para o desenvolvimento de ontologias leves através de um conjunto de termos conectados a partir de relacionamentos semânticos limitados.

Seguindo essa lógica, Gruber (2005) apresenta uma proposta denominada “TagOntology" cujo objetivo é desenvolver uma ontologia dedicada a formalizar a ação de etiquetagem a partir dos seguintes elementos: o objeto (item informacional etiquetado); as etiquetas utilizadas na marcação; o usuário que realizou a etiquetagem; e o domínio no qual a etiquetagem é realizada.

Já Specia e Motta (2007) desenvolveram um sistema que aplica uma sequência de tratamentos semânticos às Folksonomias, dentre eles: busca de tags equivalentes; agrupamento de etiquetas semelhantes com base em cálculos de medidas de similaridade de acordo com as etiquetas. Para tanto, são empregadas estratégias de pré-processamento superficiais e técnicas estatísticas, juntamente com ferramentas ontológicas fornecidas pela web semântica. Os resultados das análises realizadas por esses autores geraram clusters com tags altamente relacionadas e correspondentes a conceitos em ontologias, apresentando relacionamentos semânticos significativos.

Alruqimi e Aknin (2019), por sua vez, apresentam um algoritmo que gera uma ontologia específica de domínio a partir da Folksonomia. O algoritmo proposto por esses últimos autores inicialmente realiza a coleta de uma terminologia específica de domínio gerada pela Folksonomia, em seguida descobre um conjunto predefinido de relacionamentos conceituais explícitos entre as terminologias de domínio.

Concorda-se com Qin, Yaxi e Mou (2019), quando os autores ressaltam que a Folksonomia pode ser enriquecida semanticamente por outros Sistemas de Organização do Conhecimento (como taxonomias e ontologias) com base na estrutura sistematizada ou nas ferramentas da web semântica para auxiliar na representação e navegação, bem como sugerir aos usuários os termos semanticamente relacionados às suas consultas.

Isso se dá pelo fato de que a Folksonomia consiste em um sistema colaborativo de representação do conhecimento que reflete a mais recente terminologia em vários domínios do 
conhecimento, além de conter etiquetas que se referem a instâncias específicas (como por exemplo nomes de lugares, pessoas, fenômenos, datas etc.), podendo contemplar variadas relações semânticas entre as tags (subsunção, disjunção, meronímia, entre outras).

As taxonomias são Sistemas de Organização do Conhecimento amplamente aplicados em estruturas de navegação de sites e diretórios para a recuperação de conteúdos. Contudo, essas ferramentas têm apresentado limitações no que concerne às nuances culturais e nomenclaturas utilizadas em sua configuração. Com base nessa perspectiva, autores como Kiu e Tsui (2010) constatam que a Folksonomia e a taxonomia, em conjunto, contribuem para a melhoria da navegação e personalização da busca e recuperação do conhecimento. Para tanto, propõem um algoritmo que integra uma classificação híbrida com a taxonomia e a Folksonomia utilizando métodos heurísticos de mineração de dados, objetivando o aprimoramento na navegação do conhecimento.

Portanto, depreende-se que algumas propostas na literatura se direcionam para a criação de instrumentos de controle terminológico a partir dos dados gerados pela Folksonomia. Por outro lado, há inovações que conectam as Folksonomias a instrumentos de controle terminológico pré-existentes. Em ambos os casos é possível identificar uma unidirecionalidade, em que um sistema enriquece o outro. Todavia, verificou-se que a proposta de Alves (2012) contempla uma perspectiva bidirecional, em que ontologias e Folksonomias são combinadas em uma nova entidade denominada "Ontologia folksonomizada", harmonizando aspectos complementares de ambas.

Além das melhorias constatadas nas práticas de indexação e recuperação da informação, o autor supracitado propôs uma técnica chamada 3E Steps com vistas a prestar suporte ao desenvolvimento de ontologias a partir de dados provenientes da Folksonomia. Também foi implementado um protótipo de uma ferramenta que objetiva a construção de ontologias folksonomizadas e suporte à revisão de ontologias.

Em linhas gerais, observa-se que as propostas anteriormente descritas são relevantes para o desenvolvimento de ambientes colaborativos, uma vez que os seus métodos ou algoritmos contribuem para a criação de ferramentas mais precisas cujo objetivo é melhorar as experiências de navegação, representação, busca e recuperação semântica dos registros do conhecimento. Essas propostas podem ser integradas a modelos colaborativos de indexação, os quais de acordo com Santos (2016) são modelos que delineiam atividades colaborativas de 
indexação a serem executadas por usuários no processo de representação colaborativa da informação e servem como referências para o desenvolvimento de sistemas colaborativos, podendo ser adaptados ou aperfeiçoados em diversos contextos.

\section{CONSIDERAÇÕES FINAIS}

Em linhas gerais, verificou-se que a sinergia da Folksonomia e dos instrumentos de controle terminológico pode trazer benefícios para os sistemas colaborativos de representação e recuperação da informação no contexto do grande volume de dados e da web semânticaextensão da web atual cujos conteúdos recebem significados bem definidos- possibilitando que as pessoas e máquinas possam trabalhar em cooperação com vistas a recuperação semântica dos objetos informacionais.

É importante ressaltar que os sistemas colaborativos contemplam diferentes estruturas e modelos que refletem nas ações de etiquetagem a serem realizadas pelos usuários, bem como nos instrumentos de representação do conhecimento gerados a partir da integração da Folksonomia com outros SOCs, são eles: a) uso simultâneo e independente da Folksonomia com outro(s) SOC, considerando as suas especificidades e configurações; b) atualização contínua de instrumentos de controle terminológicos a partir da Folksonomia; c) utilização de instrumento de controle terminológico como auxílio para as práticas de etiquetagem (por meio de listas suspensas, recurso autocomplete, visualização em árvores, dentre outras formas); d) possibilidade de usuários estabelecer espontaneamente uma relação estrutural entre as tags para posterior construção automática de SOCs (ontologias ou taxonomias) a partir de agrupamentos ou algoritmos estatísticos.

Diante da capacidade e versatilidade da Folksonomia para integrar outros Sistemas de Organização do Conhecimento, bem como da possibilidade de ser enriquecida semanticamente por instrumentos como ontologias, foi possível visualizar propostas de Sistemas híbridos de Organização do Conhecimento. Esses são conceituados nesta pesquisa como Sistemas híbridos de Organização do Conhecimento- os quais agregam as potencialidades da Folksonomia e de outros instrumentos (ontologia, taxonomia, tesauro, por exemplo), podendo ser aplicados em domínios específicos, objetivando a produção significativa de metadados semânticos para fins de representação e recuperação de conteúdos na web. 
Essas propostas exploram as potencialidades da Folksonomia com o suporte dos formalismos e métodos utilizados na construção de instrumentos de controle terminológicos, descortinando novas perspectivas para auxiliar a representação e a troca de conhecimentos em ambiente web.

Portanto, acredita-se que as reflexões e proposições apresentadas nesta pesquisa podem contribuir para orientar pesquisadores e desenvolvedores de sistemas a reconhecer fatores relevantes para projetar modelos e sistemas híbridos de organização do conhecimento. Esta pesquisa também contribui para preencher as lacunas de estudos nacionais sobre a integração da Folksonomia com outros tipos de SOCs sob diferentes vieses, estabelecendo bases sólidas para identificar futuras direções de pesquisa no campo da Organização e Representação do Conhecimento.

\section{REFERÊNCIAS}

ADLER, M. Transcending Library Catalogs: A comparative study of Controlled terms in Library of Congress Subjects Headings and User-Generated Tags in Libraything for transgender Books. Journal of web librarianship, [s. l.], v. 3, n. 1, p. 309-331, 2009.

ALRUQIMI, M.; AKNIN, N. Bridging the gap between the social and semantic web: extracting domain-specific ontology from Folksonomy. Journal of Kin Saud UniversityComputer and Information Sciences, [s. l.], v. 31, n. 1, p. 15-21, Jan. 2019.

ALVES, H. A. Ontologias folksonomizadas: uma abordagem para a fusão de ontologias e folksonomias. 2012. Dissertação (Mestrado em Ciências da Computação) - Universidade Estadual de Campinas, Campinas, 2012.

AQUINO, M. A.; SANTANA, V. A. Práticas de organização e representação da informação étnico-racial em bibliotecas universitárias: necessidade de preservação da memória de negros. RICI: Revista Ibero-americana de Ciência da Informação, Brasília, v. 6, n. 2, p. 17-36, ago./dez. 2013.

BARITÉ, M. La garantia literária como herramenta de revisión de sistemas de organización del conocimiento: modelo y aplicación. 2011. Tese (Doutorado em Informação Científica) - Faculdad de Comunicación y Documentación, Universidade de Granada, Granada, 2011.

BEGHTOL, C. Bibliographic Classification Theory and Text Longuistics: Aboutness Analysis, Intertextuality and the Cognitive Act of Classifying. Journal of Documentation, Bradford, v. 42, n. 2, p. 84-113, 1986. 
CORREAA, R. F.; SANTOS, R. F. Análise das definições de Folksonomia: em busca de uma síntese. Perspectivas em Ciência da Informação, Belo Horizonte, v. 23, n. 2, p. 1-32, abr./jun. 2018.

GOLDER, S.; HUBERMAN, B. A. The structure of collaborative tagging systems. 2005. Disponível em:

https://www.researchgate.net/publication/1958429_The_Structure_of_Collaborative_Tagging _Systems. Acesso em: 02 set. 2021.

GRUBER, T. Ontology of Folksonomy: A Mash-up of Apples and Oranges. In:

CONFERENCE ON METADATA AND SEMANTICS RESEARCH (MTSR), 2005. [S. l.].

Proceedings [...]. [S. l.]: Rinton, 2005.

GUEDES, R. M. O princípio da garantia semântica e os estudos da linguagem. 2016. Tese (Doutorado em Ciência da Informação) - Universidade Federal de Minas Gerais, Belo Horizonte, 2016.

HALL, S. Cultura e representação. Rio de Janeiro: Ed. PUC-Rio: Apicuri, 2016.

KIU, C.; TSUI, E. TaxoFolk: A hybrid taxonomy-folksonomy classification for enhanced knowledge navigation. Knowledge Management Research \& Practice, [s. l.], v. 8, n. 1, p. 24-32, 2010.

KWASNIK, B. H. Semantic Warrant: a pivotal concept for our field. Knowledge Organization, [s. l.], v. 37, n. 2, p. 106-110, Apr. 2010.

LIMPENS, F.; GANDON, F.; BUFFA, M. Linking folksonomies and ontologies for supporting knowledge sharing: a state of the art. [S. l.: s. n.], 2009.

MANYIKA, J. et al. Big data: The next frontier for innovation, competition, and productivity. [S. l.]: McKinsey Global institute, 2011.

MOREIRA, W. Sistemas de Organização do Conhecimento: aspectos teóricos, conceituais e metodológicos. 2018. Tese (Livre-Docência em Sistemas de Organização do Conhecimento) - Faculdade de Filosofia e Ciências, Universidade Estadual Paulista "Júlio de Mesquita Filho”. São Paulo, 2018.

MORVILLE, P. Ambient findability. Sebastopol: O’Really, 2005.

OLSON, H. A. The power to name: representation in library catalogs. Journal of Women in Culture and Society, [s. l.], v. 26, n. 1, p. 639-668, 2001.

QIN, C.; YAXI, L.; MOU, J. User adoption of a hybrid social tagging approach in an online knowledge community. Aslib Journal of Information Management, [s. l.], v. 71, n. 2, 2019. 
QUINTARELLI, E. Folksonomies: power to the people. In: ISKO Italy-UniMIB meeting. 2005, Milán. Proceedings [...], Milán: ISKO, 2005. Disponível em: http://www.iskoi.org/doc/folksonomies.htm. Acesso em: 20 set. 2021.

MATHES, A. Folksonomies: cooperative Classification and Communication Through Shared Metadata. [S. l.: s. n.], 2004. Disponível em: https://adammathes.com/academic/computermediated-communication/folksonomies.html. Acesso em: 20 set. 2021.

MIKA, P. Ontologies are Us: a Unified Model of Social Networks and Semantics. In: INTERNATIONAL SEMANTIC WEB CONFERENCE, 4., 2005. Galway, Ireland. Proceedings [...]. [S. l.]: Springer, 2005. p. 522-536.

SANTOS, R. F. Modelos colaborativos de indexação e a sua aplicabilidade na Base de Dados Referencial de Artigos de Periódicos em Ciência da Informação. 2016. Dissertação (Mestrado em Ciência da Informação) - Universidade Federal de Pernambuco, Recife, 2016.

SANTOS, R. F. et al. A representação colaborativa da informação e a construção de linguagens documentárias sobre diversidade de gêneros: análise das contribuições do Dicionário de Gêneros - "só quem sent epode definir". In: ENCONTRO NACIONAL DE PESQUISA EM CIÊNCIA DA INFORMAÇÃO, 18., 2017. Marília. Anais eletrônicos [...]. Marília: ANCIB, 2017.

SOLER MONREAL, C.; GIL LEIVA, I. Posibilidades y límites de los tesauros frente a otros sistemas de organización del conocimiento: folksonomías, taxonomías y ontologías. Revista Interamericana de Bibliotecología, [s. l.], v. 33, n. 2, p. 361-377, jul./dic. 2010. Disponível em: https://bit.ly/2NoVNrd. Acesso em: 21 jan. 2019.

SOLOMON, Y.; BRONSTEIN, J. Serendipity in legal information seeking behavior. Aslib Journal of Information Management, [s. l.], v. 68, n. 1, p. 112-134, 2016.

SPECIA, L.; MOTTA, E. Integrating folksonomies with the semantic web. In: EUROPEAN SEMANTIC WEB CONFERENCE: RESEARCH AND APPLICATIONS, 4., 2007, Innsbruck, Austria. Proceedings [...]. [S. l.]: Springer, 2007. p. 624-639.

SPITERI, L. F. Structure and form of folksonomy tags. The road to the public library cataloque. Webology, [s. l.], v. 4, n. 2, 2007.

YEDID, N. Introducción a las Folksonomías: definición, características y diferencias con los modelos tradicionales de indización. Información, cultura y sociedade, [s. l.], v. 1, n. 29, p. 13-26, 2013.

ZENG, M. L. Knowledge Organization Systems (KOS). Knowledge Organization, [s. l.], v. 35, n. 2/3, p. 160-182, 2008. 